\title{
General Existence Theorems for Orthonormal Wavelets, an Abstract Approach
}

\author{
By \\ Larry BagGetT*, Alan CAReY**, William Moran**, \\ and Peter OHRING***
}

\begin{abstract}
Methods from noncommutative harmonic analysis are used to develop an abstract theory of orthonormal wavelets. The relationship between the existence of an orthonormal wavelet and the existence of a multi-resolution is clarified, and four theorems guaranteeing the existence of wavelets are proved. As a special case of the fourth theorem, a generalization of known results on the existence of smooth wavelets having compact support is obtained.
\end{abstract}

\section{Introduction}

We give in this paper an abstract approach to the theory of orthonormal wavelets and multi-resolutions. Although the classical context for wavelets and multiresolutions has normally been tied to Euclidean space and Fourier analysis (see the references), we are presenting here a more general picture, whose proofs rely on abstract harmonic analysis in the form of unitary group representations and Von Neumann algebras of operators. In this manner we separate the conceptually simple algebraic ideas from the more technical analytic ones. Let us describe the general setting.

Definition. By an (internal) affine structure on a (separable) Hilbert space $H$ we shall mean a (countable discrete) group $\Gamma$ of unitary operators on $H$ and another unitary operator $\delta$ on $H$ for which: $\delta^{-1} \gamma \delta$ is an element of $\Gamma$ for every $\gamma \in \Gamma$.

Definition. We refer to the elements $\gamma$ of $\Gamma$ as translations (even though $\Gamma$ need not be abelian), refer to the operator $\delta$ as a dilation, and we call the

Communicated by H. Araki, March 28, 1994.

1991 Mathematics Subject Classifications: 42C15.

* Department of Mathematics, University of Colorado, Boulder, CO 80309-0395, USA.

** School of Information Technology, Flinders University, Adelaide, South Australia.

*** Department of Mathematics, SUNY at Purchase, Purchase, NY, 10577, USA. 
pair $(\Gamma, \delta)$ the affine structure on $H$. We do not assume that $\delta \gamma \delta^{-1}$ belongs to $\Gamma$ for every $\gamma \in \Gamma$; i.e., we do not assume that $\Gamma$ is normalized by $\delta$. Ordinarily, we expect the subgroup $\Gamma_{1}=\delta^{-1} \Gamma \delta$ to be a proper subgroup of $\Gamma$; i.e., that the index $d$ of the subgroup $\Gamma_{1}$ in $\Gamma$ will be larger than 1 , but we do not require this. For an internal affine structure to exist, the group $\Gamma$ must contain a chain of subgroups $\left\{\Gamma_{i}\right\}=\left\{\delta^{-i} \Gamma \delta^{i}\right\}$, each isomorphic to $\Gamma$, for which the index of $\Gamma_{i}$ in $\Gamma_{i-1}$ is a constant independent of $i$. Ordinarily, e.g., when $\Gamma_{1}$ is a proper subgroup, we would expect $\bigcap \Gamma_{i}=\{e\}$, but again we do not require this. Note that not every group $\Gamma$ contains such a chain of subgroups, e.g., (rigid) lattice subgroups in simple Lie groups of rank $>1$. Hence, not every countable group $\Gamma$ can be part of an internal affine structure.

Definition. Let $(\Gamma, \delta)$ be an affine structure on a Hilbert space $H$. A wavelet relative to this affine structure is a finite set $\left\{\psi_{1}, \ldots, \psi_{n}\right\}$ of vectors in $H$ such that the collection $\left\{\delta^{j}\left(\gamma\left(\psi_{i}\right)\right)\right\}$ forms an orthonormal basis of $H$, where $-\infty<j<\infty, \gamma \in \Gamma$, and $1 \leq i \leq n$. We may refer to the wavelet $\left\{\psi_{i}\right\}$ as a $(\Gamma, \delta)$-wavelet.

The classical example of an internal affine structure on a Hilbert space is where $H=L^{2}(\mathbb{R}), \Gamma$ is the group of unitary operators on $H$ determined by translations by integers, and $\delta$ is the unitary operator determined by dilation by 2 :

$$
\delta f(x)=\sqrt{2} f(2 x)
$$

Other examples can similarly be constructed using dilation by some other positive integer $a$. More generally, $H$ can be taken to be $L^{2}(X, \mu), \Gamma$ a group that is determined by some group of measure-preserving transformations of $X$, and $\Delta$ some point transformation of $X$ for which $\delta^{-1} \Gamma \delta \subseteq \Gamma$. This generalization includes such things as $H=L^{2}(G)$ for $G$ a graded nilpotent Lie group, $\Gamma$ a certain discrete subgroup of $G$, and $\delta$ a homogeneous dilation of the graded group.

Our theorems show the existence of wavelets, given certain additional (typical) hypotheses. An important related definition ([ma1] [ma2]) is the following.

Definition. Let $(\Gamma, \delta)$ be an affine structure on a Hilbert space $H$. A sequence

$$
\left\{V_{j}\right\}, \quad-\infty<j<\infty,
$$

of closed subspaces of $H$ is called a multi-resolution of $H$ if

(1) $V_{j} \subseteq V_{j+1}$ for all $j$.

(2) $\delta\left(V_{j}\right)=V_{j+1}$ for all $j$. 
(3) $\bigcup V_{j}$ is dense in $H$ and $\bigcap V_{j}=\{0\}$.

(4) The subspace $V_{0}$ is invariant under each $\gamma \in \Gamma$.

Note that, unless $H$ is the trivial 0 -dimensional Hilbert space, we must have that, for any multi-resolution $\left\{V_{j}\right\}, V_{j}$ is a proper subspace of $V_{j+1}$.

Another useful bit of notation in connection with a multi-resolution is the following. For each integer $j$, let $W_{j}$ denote the orthogonal complement of the subspace $V_{j}$ in the subspace $V_{j+1}$. It is then evident that

(1) $\delta\left(W_{j}\right)=W_{j+1}$, and

(2) $\quad H$ is the direct sum $\bigoplus_{j=-\infty}^{\infty} W_{j}$ of the subspaces $\left\{W_{j}\right\}$.

As an elementary relationship among these ideas, we have:

Proposition 0.1. Let $H, \Gamma$, and $\delta$ be as in the above. Suppose there exists $a(\Gamma, \delta)$-wavelet $\left\{\psi_{1}, \ldots, \psi_{n}\right\}$ for $H$. Then:

(1) There exists a multi-resolution of $H$.

(2) The natural representation on $H$ of the cyclic group 4 , generated by the dilation $\delta$, is equivalent to a multiple of the regular representation of $\Delta$, and that multiple is $n$ times the cardinality of $\Gamma$.

Proof. If we define $V_{j}$ to be the closed linear span of the vectors $\delta^{k}\left(\gamma\left(\psi_{i}\right)\right)$, where $-\infty<k<j, \gamma \in \Gamma$ and $1 \leq i \leq N$, then the sequence $\left\{V_{j}\right\}$ forms a multiresolution of $H$. Indeed, properties 1, 2, and 3 are immediate. We show that $V_{0}$ is invariant under $\Gamma$ by first showing that $W_{k}$ is invariant under $\Gamma$ for all $k \geq 0$. Property 4 will then follow because $V_{0}$ is the orthogonal complement of the $\Gamma$-invariant subspace $\bigoplus_{k=0}^{\infty} W_{k}$.

It follows from our definition of the sequence $\left\{V_{j}\right\}$ that $W_{k}$ is the closed linear span of the vectors $\delta^{k}\left(\gamma\left(\psi_{i}\right)\right)$, for $\gamma \in \Gamma$ and $1 \leq i \leq n$. If $\eta \in \Gamma$ and $k \geq 0$, we have

$$
\eta\left(\delta^{k}\left(\gamma\left(\psi_{i}\right)\right)\right)=\delta^{k}\left(\left(\delta^{-k} \eta \delta^{k}\right)\left(\gamma\left(\psi_{i}\right)\right)\right) \in W_{k}
$$

as desired.

Finally, for each fixed $\gamma \in \Gamma$ and each $1 \leq i \leq n,\left\{\delta^{j}\left(\gamma\left(\psi_{i}\right)\right)\right\}$ forms an orthonormal set whose closed linear span is a $\Delta$-invariant subspace $H_{\gamma, i}$ of $H$ on which the action of $\Delta$ is obviously equivalent to the regular representation of $\Delta$. Since these subspaces $\left\{H_{\gamma, i}\right\}$ are pairwise orthogonal, this proves part 2 .

The converse to part 1 of Proposition 0.1 is not valid in general, as we shall see by the example below.

Let $H=l^{2}=L^{2}(\mathbf{Z})$. For each dyadic root of unity $\lambda \in[0,1)\left(\exp \left(2 \pi i 2^{k} \lambda\right)\right.$ $=1$ for some nonnegative integer $k$ ), define a multiplication operator $\gamma_{\lambda}$ on $H$ by setting

$$
\left[\gamma_{\lambda}(f)\right](n)=\exp \left(2 \pi i 2^{n} \lambda\right) f(n), \quad f \in l^{2}(Z) .
$$


The set $\Gamma$ of all these operators $\left\{\gamma_{\lambda}\right\}$ forms a countable group of unitary operators on $H$. Finally, let $\delta$ be the bilateral shift (to the right) on $H$. That is,

$$
\left[\delta\left(\left\{c_{n}\right\}\right)\right](k)=c_{k-1} .
$$

Then the pair $(\Gamma, \delta)$ forms an internal affine structure on $H$. The index of $\Gamma_{1}$ in $\Gamma$ in this case is 2 .

Proposition 0.2. There exists no $(\Gamma, \delta)$-wavelet for the preceding example. On the other hand, there does exist a multi-resolution of $H$.

Proof. The representation of the group $\Delta$ in this example is clearly equivalent to the regular representation of $\Delta$. Since the cardinality of $\Gamma$ is not equal to 1 in this case, and the regular representation of (the abelian group) $\Delta$ is not equivalent to a proper subrepresentation of itself, it follows from part 2 of Proposition 0.1 that there can be no $(\Gamma, \delta)$-wavelet in this case.

Next, define $V_{j}$ to be the set of all elements $\left\{c_{n}\right\} \in H$ for which $c_{n}=0$ for all $n \geq j$. Obviously, the sequence $\left\{V_{j}\right\}$ is a multi-resolution of $H$. In fact, in this example, every subspace $V_{j}$ is invariant under the group $\Gamma$.

Already in this proof we see an application of representation theory to our subject.

Much of the early work on wavelets has developed from the construction of a wavelet from a given multi-resolution. See [d], [ma1], [ma2], and [me]. In the literature, such multi-resolutions have in addition been assumed to have a scaling vector.

Definition. Let $H, \Gamma$, and $\delta$ be as in the above. If $\left\{V_{j}\right\}$ is a multiresolution of $H$, then $\left\{V_{j}\right\}$ is said to have a scaling vector if there exists a vector $\phi \in V_{0}$ such that the collection $\{\gamma(\phi)\}$ for $\gamma \in \Gamma$ forms an orthonormal basis of $V_{0}$.

Remark. From our abstract point of view, the existence of a scaling vector is the assumption that the representation on $V_{0}$ of $\Gamma$ is equivalent to its left regular representation. In addition, if $\phi$ is a scaling vector for the multi-resolution $\left\{V_{j}\right\}$, then $\phi$ is a cyclic vector for the representation on $H$ of the group $G$ generated by $\Gamma$ and $\delta$.

In the preceding example, there is clearly a cyclic vector (the sequence $\left\{c_{n}\right\}$ for which $c_{0}=1$ and $c_{n}=0$ otherwise), but there is no scaling vector. One can see this directly, or we can conclude it as a consequence of Theorem 1 below. In any event, we see that not every multi-resolution has a scaling vector. Moreover, it is easy to construct $(\Gamma, \delta)$-wavelets for which the multi-resolution constructed as in Proposition 0.1 has no scaling vector. Our first theorem (proved in the next section) is a generalization of various previous results in the literature. 
Theorem 1. Let $(\Gamma, \delta)$ be an internal affine structure on a separable Hilbert space $H$. Suppose $\left\{V_{j}\right\}$ is a multi-resolution of $H$ for which there exists a scaling vector $\phi$. Assume, in addition, that the subgroup $\Gamma_{1}=\delta^{-1} \Gamma \delta$ is of finite index $d$ in $\Gamma$. Then $d>1$, and there exists $a(\Gamma, \delta)$-wavelet $\psi_{1}, \ldots, \psi_{d-1}$ for $H$.

Of course, the typical problems of interest in this subject have been in constructing wavelets $\left\{\psi_{i}\right\}$ that have certain smoothness or decay properties. See [d] and [me]. Our first theorem only guarantees $\left(L^{2}\right)$ vectors with no additional attributes, but our second and third theorems address the more subtle question.

Fix an internal affine structure $(\Gamma, \delta)$ on a separable Hilbert space $H$. Now, in addition, suppose that the Hilbert space $H$ contains a dense subspace $D$ that we think of as a space of distinguished (e.g., smooth or decaying) vectors. In our context, it is reasonable to assume that $D$ is invariant under $\Gamma, \delta$, and $\delta^{-1}$. We seek a wavelet $\left\{\psi_{i}\right\}$ for $H$ consisting of vectors from $D$. We call such a wavelet a smooth wavelet. As might be expected when dealing with questions of smoothness, the situation is now considerably more technical. We make the following natural definitions.

Definition. Let $H, \Gamma, \delta$, and $D$ be as in the above. A sequence $\left\{D_{j}\right\}$ of subspaces of $D$ is called a smooth multi-resolution if it satisfies the following:

(1) $D_{j} \subseteq D_{j+1}$ for all $j$.

(2) $\delta\left(D_{j}\right)=D_{j+1}$ for all $J$.

(3) $\bigcup D_{j}=D$ is dense in $H$ and $\bigcap D_{j}=\{0\}$.

(4) $D_{0}$ is invariant under $\Gamma$.

(5) The sequence $\left\{V_{j}\right\}=\left\{\overline{D_{j}}\right\}$ is a multi-resolution of $H$, and $D_{j}=D \cap V_{j}$ for all $j$.

The smooth multi-resolution is said to have a scaling vector if there exists a vector $\phi \in D_{0}$ such that the set $\{\gamma(\phi)\}$, for $\gamma \in \Gamma$, forms an orthonormal set whose linear span is dense in $D_{0}$.

As we will necessarily be dealing with incomplete inner product spaces, we introduce the following nomenclature. Suppose $G$ is a group of inner product preserving linear transformations on an inner product space $Y$. A $G$-subspace of $Y$ is a subspace that is invariant under all elements of $G$. A linear transformation $U$ from a $G$-subspace $S$ of $Y$ into a subspace $S^{\prime}$ of $Y$ is called a $G$-morphism if it is inner product preserving and if it commutes with each $g \in G$. Two $G$-subspaces $S$ and $S^{\prime}$ of $Y$ are called G-equivalent if there exists a $G$-morphism of $S$ onto $S^{\prime}$.

Now, assume that $\left\{D_{j}\right\}$ is a smooth multi-resolution having a scaling vector $\phi$, and let $A$ denote the linear subspace of $L^{2}(\Gamma)$ consisting of the set of functions $c(\gamma)$ for which

$$
\sum_{\gamma \in \Gamma} c(\gamma) \gamma(\phi) \in D_{0}
$$


Note that, since $\phi \in D_{0}$, and $D_{0}$ is invariant under $\Gamma$, the subspace $A$ contains every function $c$ that has finite support.

Theorem 2. Let $H, \Gamma, \delta, D,\left\{D_{j}\right\}$, and $A$ be as in the above. Assume that $\left\{D_{j}\right\}$ has a scaling vector $\phi$, and define a function $h \in A$ on $\Gamma$ by

$$
\delta^{-1}(\phi)=\sum_{\gamma \in \Gamma} h(\gamma) \gamma(\phi) .
$$

As in Theorem 1, assume that the index of the subgroup $\Gamma_{1}=\delta^{-1} \Gamma \delta$ in $\Gamma$ is a finite integer $d$. Suppose further that

(1) $A$ satisfies an hereditary property with respect to $\Gamma_{1}$; namely, if $c \in A$ then $\left.c\right|_{\Gamma_{1}} \in A$.

(2) $A$ is a subspace of $L^{1}(\Gamma)$, and $c * h \in A$ whenever $c \in A$.

(3) The space $D_{0}$ satisfies the following complementation property: If $S$ is a closed $\Gamma$-subspace of $D_{0}$, then $D_{0}$ is the direct sum $S \oplus D_{0} \cap S^{\perp}$.

(4) The action of $\Gamma$ on $D_{0}$ satisfies the following cancellation property: If $S_{1}$ and $S_{2}$ are closed $\Gamma$-subspaces of $D_{0}$ and $U$ is a $\Gamma$-morphism of $S_{1}$ onto $S_{2}$, then there exists a $\Gamma$-morphism $U^{\prime}$ of $D_{0} \cap S_{1}^{\perp}$ onto $D_{0} \cap S_{2}^{\perp}$.

(5) Suppose $U$ is a $\Gamma$-morphism of $D_{0}$ onto a subspace $S$ of $D_{1}$. Suppose $T$ is a continuous, injective, linear transformation from a $\Gamma$-subspace $S^{\prime}$ of $D_{0}$ into $D_{1}$, that $T$ commutes with the action of $\Gamma$, and that $S \cap T\left(S^{\prime}\right)=\{0\}$. Then $S^{\prime}$ and $T\left(S^{\prime}\right)$ are $\Gamma$-equivalent subspaces of $D_{1}$.

Then $d>1$, and there exists a smooth wavelet $\psi_{1}, \ldots, \psi_{d-1}$ for $H$; i.e., each $\psi_{i}$ is an element of $D$.

While condition 5 of Theorem 2 appears to be somewhat artificial, it is closely related to the Gramm-Schmidt arguments used by many authors in their constructions of wavelets. It could be replaced by a stronger and cleaner condition, but it represents the minimum that appears to be required here. We discuss this and other matters in the final section.

Theorem 2 is proved in Section 2. Its hypotheses are quite delicate, but they are satisfied for example in the classical case cited earlier when $D_{0}$ consists of all finite linear combinations of translates by integers of the scaling vector. In fact, using Theorem 2 , it is possible to prove a straightforward result in the case where $\Gamma$ is abelian. Thus, if $\Gamma$ is abelian, we call a subspace $A$ of $L^{1}(\Gamma)$ almost analytic if whenever $f \in A$ and $\hat{f}$ vanishes on a set of positive Haar measure in the dual group $\hat{\Gamma}$, then $f$ is identically zero. It is perhaps worth noting that quasi-analytic functions on the circle have this property. (See $[\mathrm{m}]$ Ch. VIII.)

Theorem 3. Let $(\Gamma, \delta)$ be an affine structure on a separable Hilbert space $H$, and assume that $\Gamma$ is abelian. Suppose $\left\{D_{j}\right\}$ is a smooth multi-resolution (relative to a given dense subspace $D$ ) of $H$ that has a scaling vector $\phi$, and assume that the associated subspace $A$ is almost analytic, a selfadjoint subalgebra 
of $L^{1}(\Gamma)$, and closed under complex conjugation. Suppose that $A$ satisfies hypothesis 1 of Theorem 2, and as in Theorems 1 and 2 suppose that the index of the subgroup $\Gamma_{1}=\delta^{-1} \Gamma \delta$ in $\Gamma$ is a finite integer $d$. Then $d>1$, and there exists a $(\Gamma, \delta)$ wavelet $\psi_{1}, \ldots, \psi_{d-1}$ for $H$ for which each $\psi_{i} \in D=\bigcup D_{j}$.

Remark. The hereditary property (hypothesis 1 of Theorem 2) is automatically satisfied in the case of Theorem 3 if the algebra $A$ is closed under multiplication by characters of $\Gamma$. This is so because $\Gamma_{1}$ has finite index in $\Gamma$. Theorem 3 has a corollary when $\delta^{-1}(\phi)$ is a finite linear combination of $\Gamma$-translates of $\phi$. This special situation is one in which smooth wavelets having compact support have been constructed.

Theorem 4. Let $(\Gamma, \delta)$ be an affine structure on a separable Hilbert space $H$, and assume that $\Gamma$ is abelian and torsion free. Suppose $\left\{V_{j}\right\}$ is a multiresolution that has a scaling vector $\phi$, and that $\delta^{-1}(\phi)$ is a finite linear combination of $\Gamma$-translates of $\phi$. As in Theorems 1 and 2, suppose that the index of the subgroup $\Gamma_{1}=\delta^{-1} \Gamma \delta$ in $\Gamma$ is a finite integer $d$. Then $d>1$, and there exists $a(\Gamma, \delta)$ wavelet $\psi_{1}, \ldots, \psi_{d-1}$ for $H$ for which each $\psi_{i}$ is a finite linear combination of vectors of the form $\delta(\gamma(\phi))$.

\section{$\S 1$. Proof of Theorem 1}

In this section we prove Theorem 1. We derive it as a consequence of a sequence of lemmas.

Lemma 1.1. The action of the group $\Gamma$ on the subspace $V_{0}$ is equivalent to the left regular representation $\Lambda$ of $\Gamma$.

Proof. Since $\phi$ is a scaling vector, there is an obvious identification of $V_{0}$ and $L^{2}(\Gamma)$, which is determined by the map $\gamma(\phi) \rightarrow \chi_{\{\gamma\}}$. This identification clearly effects a unitary equivalence between the action of $\Gamma$ on $V_{0}$ and the representation $\Lambda$.

Lemma 1.2. The action of the group $\Gamma$ on the subspace $V_{1}$ is equivalent to a direct sum of $d$ copies of $\Lambda$.

Proof. It follows from our assumptions that the index of the subgroup $\Gamma$ in the group $\Gamma^{1}=\delta \Gamma \delta^{-1}$ is $d$. Also, because $\phi$ is a scaling vector and $\delta$ is unitary, we have that the collection $\left\{\gamma^{1}(\delta(\phi))\right\}$, as $\gamma^{1}$ runs over $\Gamma^{1}$, forms an orthonormal basis of $V_{1}$. If $\eta_{0}, \ldots, \eta_{d-1}$ are coset representatives for the $d$ distinct (right) cosets of $\Gamma$ in $\Gamma^{1}$, then we may decompose the space $V_{1}$ as the direct sum of $d$ subspaces $N_{0}, \ldots, N_{d-1}$ where $N_{i}$ is the set of all elements of $V_{1}$ of the form $\sum_{\gamma \in \Gamma} c_{\gamma} \gamma\left(\eta_{i}(\delta(\phi))\right)$. Clearly, the action of $\Gamma$ on each $N_{i}$ is equivalent to the left regular representation $\Lambda$ of $\Gamma$, and this completes the proof of the lemma. 
Remark. A more sophisticated argument showing Lemma 1.2, but an argument more in line with the spirit of this article, goes as follows. As in the proof of Lemma 1.1, observe that the action of $\Gamma^{1}$ on $V_{1}$ is equivalent to the left regular representation $\Lambda^{1}$ of $\Gamma^{1}$. Hence, the action of $\Gamma$ on $V_{1}$ is equivalent to the restriction to $\Gamma$ of $\Lambda^{1}$. But this restriction is known to be equivalent to $d \times \Lambda$. The next lemma is the deep part of the proof of Theorem 1. It relies on the finiteness of the Von Neumann algebra of $\Gamma$. Specifically, the way in which this finiteness property is used is in the following cancellation property:

Cancellation Property。 Let $G$ be a locally compact group, and let $\rho$ be a unitary representation of $G$ whose commutant is a finite Von Neumann algebra. Suppose that $\rho$ is equivalent to $\sigma_{1} \oplus \sigma_{2}$, and also $\rho$ is equivalent to $\sigma_{1} \oplus \sigma_{3}$. Then $\sigma_{2}$ is equivalent to $\sigma_{3}$.

(See 3.2.3 Prop. 6 of $[\mathrm{dx}]$.) (Note that the cancellation property fails in general, for example when the left regular representation of $G$ is equivalent to an infinite multiple of itself.) We shall apply this cancellation property to a representation $\rho$ that is a direct sum of $d$ copies of $\Lambda$. Because $\Gamma$ is discrete, the commutant of $\Lambda$, which is the Von Neumann algebra $\operatorname{VN}(\Gamma)$ is finite, whence the commutant of $\rho$ is finite as well.

Lemma 1.3. The index $d$ must be larger than 1, and the action of $\Gamma$ on the orthogonal complement $W_{0}$ of $V_{0}$ in $V_{1}$ is equivalent to a direct sum of $d-1$ copies of $\Lambda$.

Proof. Write $\rho$ for the representation of $\Gamma$ determined by its action on $V_{1}$. Then, by Lemma $1.2, \rho$ is equivalent to a direct sum of $d$ copies of 1. (Note that if $d$ were 1 then $\rho$ would be equivalent to 1 .) If $\sigma$ denotes the representation of $\Gamma$ determined by its action on $W_{0}$, then we have also that $\rho$ is equivalent to $\Lambda \oplus \sigma$. Hence, by the cancellation property, we have that $\sigma$ is equivalent to a direct sum of $d-1$ copies of $\Lambda$. Moreover, since $V_{0}$ is a proper subspace of $V_{1}$, we have that $W_{0} \neq\{0\}$, and $\sigma$ is a nonzero representation. This implies that $d>1$, and the lemma is proved.

We now complete the proof of Theorem 1 as follows. Write $W_{0}$ as a direct sum $\bigoplus_{i=1}^{d-1} M_{i}$ of subspaces $\left\{M_{i}\right\}$ on which the action of $\Gamma$ is equivalent to $\Lambda$, and denote by $U_{i}: M_{i} \rightarrow L^{2}(\Gamma)$ a unitary operator that effects this equivalence. For each $1 \leq i \leq d-1$, let $\psi_{i}=U_{i}^{-1}\left(\chi_{\{e\}}\right)$. Then, the collection $\left\{\gamma\left(\psi_{i}\right)\right\}$ forms an orthonormal basis for the subspace $M_{i}$. Hence, the collection $\left\{\gamma\left(\psi_{i}\right)\right\}$, for $\gamma \in \Gamma$ and $1 \leq i \leq d-1$, forms an orthonormal basis for the subspace $W_{0}$. But then the collection $\left\{\delta^{j}\left(\gamma\left(\psi_{i}\right)\right)\right\}$, for $-\infty<j<\infty, \gamma \in \Gamma$, and $1 \leq i \leq$ $d-1$, forms an orthonormal basis for $\bigoplus W_{j}$ which is $H$. Therefore, the set $\psi_{1}, \ldots, \psi_{d-1}$ is a $(\Gamma, \delta)$-wavelet, as desired. 


\section{§ 2. Proofs of Theorems 2, 3, and 4}

First we prove Theorem 2. We argue to some degree along the lines of the proof of Theorem 1, although we will be forced here to deal with inner product spaces that are not necessarily complete. Note that the cancellation property, so important in the proof of Theorem 1, is already evident in hypothesis 4 of Theorem 2.

It follows from hypotheses 3,4 , and 5 , by applying $\delta^{-1}$ to $D_{0}$, that the inner product space $D_{-1}$ satisfies:

(1) If $S$ is a closed $\Gamma_{1}$-subspace of $D_{-1}$, then $D_{-1}$ is the direct sum $S \oplus D_{-1} \cap S^{\perp}$.

(2) Suppose $S_{1}$ and $S_{2}$ are closed $\Gamma_{1}$-subspaces of $D_{-1}$ and that $U$ is a $\Gamma_{1}$-morphism of $S_{1}$ onto $S_{2}$; i.e., $S_{1}$ and $S_{2}$ are $\Gamma_{1}$-equivalent. Then there exists a $\Gamma_{1}$-morphism $U^{\prime}$ of $D_{-1} \cap S_{1}^{\perp}$ onto $D_{-1} \cap S_{2}^{\perp}$; i.e., $D_{-1} \cap S_{1}^{\perp}$ and $D_{-1} \cap S_{2}^{\perp}$ are $\Gamma_{1}$-equivalent.

(3) Suppose $U$ is a $\Gamma_{1}$-morphism of $D_{-1}$ onto a subspace $S$ of $D_{0}$. Suppose $T$ is a continuous, injective, linear transformation from a $\Gamma_{1}$ subspace $S^{\prime}$ of $D_{-1}$ into $D_{0}$, that $T$ commutes with the action of $\Gamma_{1}$, and that $S \cap T\left(S^{\prime}\right)=\{0\}$. Then $S^{\prime}$ and $T\left(S^{\prime}\right)$ are $\Gamma_{1}$-equivalent subspaces of $D_{0}$.

Next, let $\eta_{0}, \ldots, \eta_{d-1}$ be a set of coset representatives for the right cosets $\left(\Gamma_{1} \eta\right)$ in $\Gamma$, and assume that $\eta_{0}$ is the identity operator $I$ on $H$. For each $0 \leq i \leq d-1$ let $K_{i}$ be the subspace of $D_{0}$ consisting of the elements

$$
\sum_{\gamma_{1} \in \Gamma_{1}} c\left(\gamma_{1}\right) \gamma_{1}\left(\eta_{i}(\phi)\right)
$$

where the function $c$ belongs to the subspace $A$. It follows from hypothesis 1 of Theorem 2 that $D_{0}$ is the direct sum $D_{0}=\bigoplus_{i=0}^{d-1} K_{i}$. Moreover, the map $W_{i}$, defined on $K_{i}$ into $K_{0}$ by

$$
W_{i}\left(\sum_{\gamma_{1} \in \Gamma_{1}} c\left(\gamma_{1}\right) \gamma_{1}\left(\eta_{i}(\phi)\right)\right)=\sum_{\gamma_{1} \in \Gamma_{1}} c\left(\gamma_{1}\right) \gamma_{1}(\phi),
$$

is a $\Gamma_{1}$-morphism of $K_{i}$ onto $K_{0}$.

Next, let $h(\gamma)$ be the function on $\Gamma$ for which

$$
\delta^{-1}(\phi)=\sum_{\gamma \in \Gamma} h(\gamma) \gamma(\phi)
$$

Observe that $h(\eta)=\left(\delta^{-1}(\phi), \eta(\phi)\right)$, so that if $\gamma_{1}, \gamma_{2} \in \Gamma_{1}$, then

$$
\sum_{\eta \in \Gamma} h\left(\gamma_{1} \eta\right) \overline{h\left(\gamma_{2} \eta\right)}=1
$$

if $\gamma_{1}=\gamma_{2}$ and is 0 otherwise. Consequently, making use of hypothesis 2 of 
the theorem, the map $W$ defined by

$$
\begin{aligned}
W\left(\sum_{\gamma_{1} \in \Gamma_{1}} c\left(\gamma_{1}\right) \gamma_{1}(\phi)\right) & =\sum_{\eta \in \Gamma} \sum_{\gamma_{1} \in \Gamma_{1}} c\left(\gamma_{1}\right) h\left(\gamma_{1}^{-1} \eta\right) \eta(\phi) \\
& =\sum_{\eta \in \Gamma} c * h(\eta) \eta(\phi)
\end{aligned}
$$

is a $\Gamma_{1}$-morphism of $K_{0}$ onto $D_{-1}$. Indeed, $W^{-1}$ is given by the formula

$$
W^{-1}\left(\sum_{\eta \in \Gamma} d(\eta) \eta(\phi)\right)=\sum_{\gamma_{1} \in \Gamma_{1}} d * h^{*}\left(\gamma_{1}\right) \gamma_{1}(\phi) .
$$

We will use the following lemma, in which the subspace $M_{0}$ will correspond to our subspace $D_{-1}$, and where the unitary operators $U_{i}$ will correspond to our operators $W \circ W_{i}$.

Lemma 2.1. Let $D_{0}$ be an inner product space, and let $\Gamma_{1}$ be a group of inner product preserving linear transformations of $D_{0}$ onto itself. Suppose $D_{0}$ is the orthogonal (algebraic) direct sum $M_{0} \oplus M_{0}^{\perp}$, where $M_{0}$ is a $\Gamma_{1}$-invariant, closed subspace of $D_{0}$ that satisfies the following properties.

(1) If $S$ is a closed $\Gamma_{1}$-subspace of $M_{0}$, then $M_{0}$ is the direct sum $S \oplus M_{0} \cap$ $S^{\perp}$.

(2) If $S_{1}$ and $S_{2}$ are closed $\Gamma_{1}$-subspaces of $M_{0}$ and $U$ is a $\Gamma_{1}$-morphism of $S_{1}$ onto $S_{2}$, then there exists a $\Gamma_{1}$-morphism $U^{\prime}$ of the $\Gamma_{1}$-subspace $M_{0} \cap S_{1}^{\perp}$ onto $M_{0} \cap S_{2}^{\perp}$.

(3) Suppose $U$ is a $\Gamma_{1}$-morphism of $M_{0}$ onto a subspace $S$ of $D_{0}$. Suppose $T$ is a continuous, injective, linear transformation from a $\Gamma_{1}$-subspace $S^{\prime}$ of $M_{0}$ into $D_{0}$, that $T$ commutes with the action of $\Gamma_{1}$, and that $S \cap T\left(S^{\prime}\right)=\{0\}$. Then $S^{\prime}$ and $T\left(S^{\prime}\right)$ are $\Gamma_{1}$-equivalent subspaces of $D_{0}$.

Suppose $d$ is an integer $\geq 1$ and that $K_{0}, \ldots, K_{d-1}$ are pairwise orthogonal closed $\Gamma_{1}$-subspaces of $D_{0}$ for which $D_{0}$ is the direct sum $\bigoplus_{i=0}^{d-1} K_{i}$ and that for each $0 \leq i \leq d-1$ there exists a $\Gamma_{1}$-morphism $U_{i}$ of $K_{i}$ onto $M_{0}$.

Then there exist pairwise orthogonal, $\Gamma_{1}$-invariant, closed subspaces $M_{1}, \ldots$, $M_{d-1}$ of $D_{0}$, each orthogonal to $M_{0}$, such that for each $1 \leq i \leq d-1$ there exists a $\Gamma_{1}$-morphism $V_{i}$ of $M_{i}$ onto $K_{i}$.

Remark. Notice that we do not assert that $D_{0}$ is the direct sum of the subspaces $\left\{M_{i}\right\}$. Indeed, this need not be the case in this generality. However, we will want this direct sum decomposition to hold in our case, but to obtain it we will again need to exploit the cancellation property of Section 1, i.e., the finiteness of $\mathrm{VN}(\Gamma)$.

Proof. We argue by induction on $d$, and we note that the case $d=1$ is vacuously true. Assume then that $d>1$. 
Case 1. Suppose that $M_{0} \cap K_{d-1}=\{0\}$. Write $D_{0}^{\prime}=\bigoplus_{i=0}^{d-2} K_{i}$ and set $M_{0}^{\prime}$ equal to $Q\left(M_{0}\right)$, where $Q$ is the orthogonal projection of $D_{0}$ onto $D_{0}^{\prime}$. Because $D_{0}=D_{0}^{\prime} \oplus K_{d-1}$, it follows that $Q$ commutes with each $\gamma_{1} \in \Gamma_{1}$ and maps $M_{0}$ continuously and injectively onto $Q\left(M_{0}\right)$. Hence, by hypothesis 3 of the lemma, there exists a $\Gamma_{1}$-morphism $U^{\prime}$ of $M_{0}$ onto $M_{0}^{\prime}$. The inductive hypotheses apply, and we let $M_{1}^{\prime}, \ldots, M_{d-2}^{\prime}$ be pairwise orthogonal closed $\Gamma_{1}$-subspaces of $D_{0}^{\prime}$, each orthogonal to $M_{0}^{\prime}$, such that for each $1 \leq i \leq d-2$ there exists a $\Gamma_{1}$-morphism $V_{i}$ of $M_{i}^{\prime}$ onto $K_{i}$. Note that for $1 \leq i \leq d-2$, we have that $M_{i}^{\prime}$ is orthogonal both to $M_{0}^{\prime}$ and to $K_{d-1}$, whence is orthogonal to the closed subspace $D^{\prime \prime}$ spanned by $K_{d-1}$ and $M_{0}$. Let $Q^{\prime}$ denote the projection of $D^{\prime \prime}$ onto $M_{0}^{\perp}$. Then $Q^{\prime}$ maps $K_{d-1}$ continuously and injectively onto a subspace $S^{\prime \prime}$ of $M_{0}^{\perp}$, whence, by composing $Q^{\prime}$ with $U_{d-1}^{-1}$, we have a continuous, injective linear transformation $T=Q^{\prime} \circ U_{d-1}^{-1}$ of $M_{0}$ onto a subspace $S^{\prime \prime}$ of $M_{0}^{\perp}$. By hypothesis 3, there exists a $\Gamma_{1}$-morphism $U^{\prime}$ of $M_{0}$ onto the subspace $S^{\prime \prime} \equiv M_{d-1}$ of $M_{0}^{\perp}$. Set $V_{d-1}=U_{d-1}^{-1} \circ U^{\prime-1}$. Since $M_{d-1}=Q^{\prime}\left(K_{d-1}\right)$ is orthogonal to each $M_{i}$ for $1 \leq i \leq d-2$, the proof of the lemma is complete for this first case.

Case 2. For each $0 \leq i \leq d-1$, define $K_{i}^{i}=K_{i} \cap M_{0}$. By condition 1 , we may write

$$
M_{0}=\bigoplus_{i=0}^{d-1} K_{i}^{i} \oplus M_{0}^{*}
$$

where $M_{0}^{*}$ is $\Gamma_{1}$-invariant and equals

$$
M_{0} \cap\left[\bigoplus_{i=0}^{d-1} K_{i}^{i}\right]^{\perp} .
$$

Using condition 2 , and the fact that there exists for each $0 \leq i \leq d-1$ a $\Gamma_{1}$-morphism $U_{i}$ of $K_{i}$ onto $M_{0}$, there exist subspaces $\left\{K_{i}^{j}\right\}, 0 \leq i, j \leq d-1$, $j \neq i$, so that we may write

$$
K_{i}=\bigoplus_{j=0}^{d-1} K_{i}^{j} \oplus K_{i}^{*}
$$

where, for each $0 \leq j \leq d-1, j \neq i$, there exists a $\Gamma_{1}$-morphism $W_{i}^{j}$ of $K_{i}^{j}$ onto $K_{j}^{j}$, and where there exists a $\Gamma_{1}$-morphism $W_{i}^{*}$ of $K_{i}^{*}$ onto $M_{0}^{*}$. Notice that the $d^{2}+d$ subspaces $\left\{K_{i}^{j}, K_{k}^{*}\right\}$, for $0 \leq i, j, k \leq d-1$, are pairwise orthogonal and $\Gamma_{1}$-invariant.

Let $D_{0}^{*}=\bigoplus_{i=0}^{d-1} K_{i}^{*}$. Because $M_{0}^{*}$ is a subspace of $D_{0}^{*}$, the hypotheses of the lemma apply to the inner product space $D_{0}^{*}$, subspace $M_{0}^{*}$, set of subspaces $\left\{K_{i}^{*}\right\}$ and $\Gamma_{1}$-morphisms $\left\{W_{i}^{*}\right\}$, and since $M_{0}^{*} \cap K_{d-1}^{*}=\{0\}$, we are in a position to use the first part, i.e., case 1, of this proof. Hence, there exist pairwise orthogonal $\Gamma_{1}$-subspaces $M_{1}^{*}, \ldots, M_{d-1}^{*}$ of $D_{0}^{*}$, each orthogonal to $M_{0}^{*}$, such that 
for each $1 \leq i \leq d-1$ there exists a $\Gamma_{1}$-morphism $V_{i}^{*}$ of $M_{i}^{*}$ onto $K_{i}^{*}$. Now, for each $1 \leq i \leq d-1$, define

$$
M_{i}=\bigoplus_{k=0}^{d-1} K_{k}^{k+i \bmod d} \oplus M_{i}^{*} .
$$

Clearly, $M_{i}$ and $M_{j}$ are orthogonal if $i \neq j$, each $M_{i}$ is orthogonal to

$$
M_{0}=\bigoplus_{k=0}^{d-1} K_{k}^{k} \oplus M_{0}^{*}
$$

and, for each $1 \leq i \leq d-1$, there exists a $\Gamma_{1}$-morphism $V_{i}$ of $M_{i}$ onto $K_{i}$. Indeed, the map $V_{i}$ is the direct product

$$
V_{i}=U_{i}^{-1} \circ\left(\prod_{k=0}^{d-1} W_{k}^{k+i \bmod d} \otimes W_{i}^{*} \circ V_{i}^{*}\right) .
$$

This completes the proof of the lemma.

Now we apply the lemma to our situation. Thus, there exist pairwise orthogonal $\Gamma_{1}$-subspaces $M_{1}, \ldots, M_{d-1}$ of $D_{0}$, each orthogonal to $D_{-1}$, such that for each $1 \leq i \leq d-1$ there exists a $\Gamma_{1}$-morphism $V_{i}$ of $M_{i}$ onto $K_{i}$. For each $1 \leq i \leq d-1$, let $\phi_{i}$ be the element of $M_{i}$ such that $V_{i}\left(\phi_{i}\right)=\eta_{i}(\phi)$. Define $\psi_{i}=\delta\left(\phi_{i}\right)$. Then each $\psi_{i} \in D$.

Lemma 2.2. The index $d$ of $\Gamma_{1}$ in $\Gamma$ is larger than 1 , and $\psi_{1}, \ldots, \psi_{d-1}$ is a $(\Gamma, \delta)$-wavelet.

Proof. That $d>1$ follows from Theorem 1, since the sequence $\left\{\overline{D_{j}}\right\}$ is a multi-resolution of $H$ that has a scaling vector. Let $N_{i}$ be the closed subspace of $V_{1}$ spanned by the vectors $\left\{\gamma\left(\psi_{i}\right)\right\}$ for $\gamma \in \Gamma$. Note that, because $\phi_{i}$ is orthogonal to the subspace $D_{-1}$, each $N_{i}$ is actually contained in $W_{0}=$ $V_{0}^{\perp} \cap V_{1}$. Then the subspaces $\left\{N_{i}\right\}$ are pairwise orthogonal, and the action of $\Gamma$ on each $N_{i}$ is equivalent to its regular representation $\Lambda$. Therefore, the action of $\Gamma$ on the direct sum $\bigoplus_{i=1}^{d-1} N_{i}$ is equivalent to $(d-1) \times \Lambda$. It follows from the cancellation property of Section 1 that $W_{0}=\bigoplus_{i=1}^{d-1} N_{i}$. Therefore, the vectors $\left\{\gamma\left(\psi_{i}\right)\right\}$, for $\gamma \in \Gamma$ and $1 \leq i \leq d-1$, span $W_{0}$, whence the vectors $\left\{\delta^{j}\left(\gamma\left(\psi_{i}\right)\right)\right\}$ span all of $H$. It follows directly that the vectors $\left\{\delta^{j}\left(\gamma\left(\psi_{i}\right)\right)\right\}$ form an orthonormal set for $-\infty<j<\infty, \gamma \in \Gamma$, and $1 \leq i \leq d-1$, and so the proof of Lemma 2.2 and hence Theorem 2 is complete.

Now we derive Theorem 3 from Theorem 2. We assume at this point that $\Gamma$ is abelian and that the subspace $A$ satisfies hypothesis 1 of Theorem 2, is a selfadjoint subalgebra of $L^{1}(\Gamma)$, consists of almost analytic functions, and is closed under complex conjugation.

Lemma 2.3. If $S$ is a nontrivial $\Gamma$-invariant subspace of $D_{0}$, then $S$ is dense in $D_{0}$. That is, there are no nontrivial, closed $\Gamma$-subspaces of $D_{0}$. 
Proof. As before, we may identify $V_{0}=\overline{D_{0}}$ with $L^{2}(\Gamma)$ and therefore $D_{0}$ with the subspace $A$. We may prove the lemma by showing that any nontrivial $\Gamma$-invariant subspace of $\hat{A}$ is dense in $\hat{A}$. Thus, let $p(\omega)$ be a member of $A$ and write $\hat{S}$ for the $\Gamma$-invariant subspace of $\hat{A}$ generated by $p$. If $h \in L^{2}(\hat{\Gamma})$ is orthogonal to $\hat{S}$, then

$$
\int_{\hat{\Gamma}} \gamma(\omega) p(\omega) \overline{h(\omega)} d \omega=0
$$

for every $\gamma \in \Gamma$. But this implies that the Fourier transform of the $L^{1}$ function $p \bar{h}$ is 0 , whence $p(\omega) \bar{h}(\omega)=0$ for almost all $\omega \in \hat{\Gamma}$. Now, because $A$ is almost analytic, we obtain that either $p=0$ or $h=0$, implying that $\hat{S}$ is dense in $L^{2}(\hat{\Gamma})$ and so is dense in $\hat{A}$ as claimed.

To complete the proof of Theorem 3, we need only show that hypothesis 5 of Theorem 2 holds, since hypotheses 3 and 4 hold vacuously in this case and hypothesis 2 is implied by our assumptions on $A$. We may write

$$
D_{1}=\bigoplus_{i=0}^{d-1} L_{i}
$$

where $L_{i}$ consists of all those $\psi \in D_{1}$ for which

$$
\psi=\sum c(\gamma) \gamma\left(\eta_{i}(\delta(\phi))\right)
$$

$c(\gamma)$ belongs to $A$, and where $\eta_{0}, \ldots, \eta_{d-1}$ are coset representatives for $\Gamma$ in $\Gamma^{1}=\delta \Gamma \delta^{-1}$. The map

$$
\sum_{\gamma} c(\gamma) \gamma(\phi) \rightarrow \sum_{\gamma} c(\gamma) \gamma\left(\eta_{i}(\delta(\phi))\right)
$$

is a $\Gamma$-morphism of $D_{0}$ onto $L_{i}$. It then follows from Lemma 2.3 that every nontrivial $\Gamma$-subspace of $L_{i}$ is dense in $L_{i}$. Observe that if

$$
\psi_{1}=\sum c_{1}(\gamma) \gamma\left(\eta_{i}(\delta(\phi))\right)
$$

and

$$
\psi_{2}=\sum c_{2}(\gamma) \gamma\left(\eta_{i}(\delta(\phi))\right)
$$

belong to $L_{i}$, it follows that the function $\theta$ defined on $\Gamma$ by

$$
\theta(\gamma)=\left(\gamma\left(\psi_{1}\right), \psi_{2}\right)
$$

belongs to the (selfadjoint, conjugate-closed algebra) $A$. Indeed,

$$
\begin{aligned}
\theta(\gamma) & =\sum_{\gamma_{1}} \sum_{\gamma_{2}} c\left(\gamma_{1}\right) \overline{c\left(\gamma_{2}\right)}\left(\gamma\left(\gamma_{1}\left(\eta_{i}(\delta(\phi))\right)\right), \gamma_{2}\left(\eta_{i}(\delta(\phi))\right)\right) \\
& =\sum_{\gamma_{1}} c_{1}\left(\gamma_{1}\right) \overline{c_{2}\left(\gamma \gamma_{1}\right)} \\
& =\overline{c_{2} * c_{1}^{*}(\gamma)} .
\end{aligned}
$$

The fact that hypothesis 5 holds follows from the next lemma. 
Lemma 2.4. If $T$ is a continuous injective linear transformation of $D_{0}$ into $D_{1}$ that commutes with the action of $\Gamma$, then there exists a $\Gamma$-morphism $U$ of $D_{0}$ onto the range of $T$.

Proof. As above, write $D_{1}=\bigoplus L_{i}$, and write $T(\zeta)=\sum T_{i}(\zeta)$ as the decomposition of $T(\zeta)$ relative to the $L_{i}$ 's. Then each $T_{i}$ is a continuous linear map of $D_{0}$ into $L_{i}$ that commutes with the action of $\Gamma$. We identify, as above, $D_{0}$ with the subspace $A$ and $V_{0}=\overline{D_{0}}$ with all of $L^{2}(\Gamma)$. Clearly, by definition, we may also identify each $L_{i}$ with the subspace $A$. It follows from Lemma 2.3 that either $T_{i}$ is injective and onto a dense subspace of $L_{i}$ or $T_{i} \equiv 0$. We claim that each $T_{i}$ is a constant multiple of a $\Gamma$-morphism $U_{i}$. This is certainly true if $T_{i} \equiv 0$. Otherwise, fix $i$, and let $T_{i}^{*}$ denote the adjoint operator of $T_{i}$. Then $T_{i}^{*}$ maps the completion $\overline{L_{i}}$ of $L_{i}$ into the completion $V_{0}$ of $D_{0}$. We show first that, because $T_{i}$ commutes with the action of $\Gamma, T_{i}^{*}$ actually maps $L_{i}$ into $D_{0}$. Indeed, if $\psi \in L_{i}$, then $T_{i}^{*}(\psi) \in L^{2}(\Gamma)$. Furthermore

$$
\begin{aligned}
{\left[T_{i}^{*}(\psi)\right](\gamma) } & =\left(T_{i}^{*}(\psi), \chi_{\{\gamma\}}\right) \\
& =\left(\gamma(\psi), T_{i}\left(\chi_{\{e\}}\right)\right)
\end{aligned}
$$

which is an element of $A \equiv D_{0}$ by the calculation above.

Therefore, $T_{i}^{*}$ maps $L_{i}$ into $D_{0}$, from which it follows that $T_{i}^{*} \circ T_{i}$ is a positive linear transformation of $D_{0}$ into itself that commutes with the action of $\Gamma$. Again using Lemma 2.3, we see that this implies that $T_{i}^{*} \circ T_{i}=p_{i} I$, on $D_{0}$ where $p_{i}$ is a positive number. Clearly then $U_{i}=\left(1 / \sqrt{p_{i}}\right) T_{i}$ is an isometry of $D_{0}$ onto the range of $T_{i}$ that commutes with the action of $\Gamma$. Now define $U: D_{0} \rightarrow D_{1}$ by

$$
U(\zeta)=\sum_{i \mid T_{i} \neq 0} U_{i}(\zeta)
$$

This completes the proof of the lemma, and therefore of Theorem 3.

Finally in this section we show that Theorem 4 is a straightforward consequence of Theorem 3. Given the hypotheses of Theorem 4, let $D_{0}$ be the subspace of $H$ consisting of the vectors $\sum_{i=1}^{n} c\left(\gamma_{i}\right) \gamma_{i}(\phi)$, and let the linear space $A$ be the set of all functions $c(\gamma)$ having finite support on $\Gamma$. It follows that $A$ is a selfadjoint subalgebra of $L^{1}(\Gamma)$ and is closed under complex conjugation. Then set $D_{j}$ equal to $\delta^{j}\left(D_{0}\right)$. This sequence $\left\{D_{j}\right\}$ is a smooth multi-resolution of $H$. Since hypothesis 1 holds by the remark following the statement of Theorem 3, all that remains to complete the proof is the observation that when $\Gamma$ is torsion-free the trigonometric polynomials (the subspace $A$ ) form an almost analytic subspace. 


\section{§3. Corollaries of the Abstract Approach}

As before, let $h$ be the function on $\Gamma$ for which

$$
\delta^{-1}(\phi)=\sum_{\gamma \in \Gamma} h(\gamma) \gamma(\phi)
$$

Introduce an $L^{2}\left(\Gamma_{1}\right)$ valued inner product on $L^{2}(\Gamma)$ by $\left\langle g_{1}, g_{2}\right\rangle_{\Gamma_{1}}=\left.g_{1} * g_{2}^{*}\right|_{\Gamma_{1}}$ for each pair $g_{1}, g_{2} \in L^{2}(\Gamma)$ where $g_{2}^{*}(\gamma)=\overline{g_{2}\left(\gamma^{-1}\right)}$. The following result is easy to check.

Lemma 3.1. The orthogonality of $g$ with $\left\{\gamma_{1} \cdot h \mid \gamma_{1} \in \Gamma_{1}\right\}$ is expressed by

$$
\langle g, h\rangle_{\Gamma_{1}}=0 \text {. }
$$

Similarly, the orthogonality of $g$ with any other $\Gamma_{1}$ submodule of $L^{2}(\Gamma)$ with cyclic vector $g_{1}$ is expressed by the condition $\left\langle g, g_{1}\right\rangle_{\Gamma_{1}}=0$.

Introduce the notation

$$
g(\phi)=\sum_{\gamma \in \Gamma} g(\gamma) \gamma(\phi)
$$

for $g \in L^{2}(\Gamma)$.

Lemma 3.2. With $D, H, \Gamma, \delta, \phi$ as in Theorem 2 the following are equivalent.

(1) There is a smooth $(\Gamma, \delta)$-wavelet.

(2) There is an inner product preserving map $U: D_{0} \rightarrow D_{0}$ commuting with the $\Gamma_{1}$-action such that $U \phi=h(\phi)$.

(3) There exist functions $g_{j}, j=1,2, \ldots, d-1$ on $\Gamma$ with $g_{j}(\phi) \in D_{0}$ such that with $g_{0}=h$,

$$
\left\langle g_{i}, g_{j}\right\rangle_{\Gamma_{1}}=\chi_{\{e\}}, \quad i, j=0,1, \ldots, d-1 .
$$

Proof. In view of our preceding results it is sufficient to show that 3 implies 2. Using the isomorphism of the closure of $D_{0}$ with $L^{2}(\Gamma)$ it is sufficient to define the required isometry on the image of $D_{0}$ in $L^{2}(\Gamma)$. If $f \in L^{2}(\Gamma)$ then $f=\sum_{i} f_{i}$ with $f_{i} \in K_{i}$. Define

$$
U f=\sum_{i} U f_{i}=\sum_{i} \eta^{-1} f_{i} * g_{i}
$$

It is straightforward to check that $U$ is an isometry.

Remark. If $\Gamma_{1}$ has index two in $\Gamma$ then these observations enable us to determine the range of $Q$ as the range of an isometry. For if $\eta \notin \Gamma_{1}$ and we let $h_{1}$ be the restriction of $h$ to the coset $\eta \Gamma_{1}$ then one easily checks that $Q\left(\delta_{\eta}\right)$ (with $\delta_{\eta}$ being the characteristic function of $\eta$ ) is the function $f=\delta_{\eta}\left(1-h_{1}^{*} h\right)$ in $L^{2}(\Gamma)$. Let $h_{0}$ denote the restriction of $h$ to $\Gamma_{1}$ then $\langle h, h\rangle_{\Gamma_{1}}=h_{0}^{*} h_{0}+h_{1}^{*} h_{1}$ 
$=1$. Substituting this relation into the definition of $f$ gives $f=\delta_{\eta}\left(h_{0}-h_{1}\right) * h_{0}$. Assuming $h_{0}$ is a function of finite support on $\Gamma_{1}$ allows us to conclude that the range of $Q$ is just $S=\left\{a * \delta_{\eta}\left(h_{0}-h_{1}\right) * \mid a \in A\right\}$. Nevertheless, even without this assumption, it is easy to see that the function $g=\delta_{\eta}\left(h_{0}-h_{1}\right)^{*}$ is orthogonal to $D_{-1}$ and satisfies $\left.\langle g, g\rangle\right|_{\Gamma_{1}}=1$ so that it gives the required wavelet in the index two case. Now suppose that $\Gamma$ is finitely generated, abelian and torsion free so that it must be a product of $n$ copies of the integers for some $n$ and hence $\Gamma / \Gamma_{1}$ is a direct product of cyclic groups. The main impediment to verifying condition 5 of Theorem 2 for the abelian case stems from the fact that we do not have a method of handling cyclic groups of order greater than two. We note however that we can reformulate the problem of verifying hypothesis 3 of Lemma 2.1 in more familiar terms.

Let $\chi_{j}, j=0,1,2, \ldots, d-1$ denote the characters in $\Gamma_{1}^{\perp}$ with $\chi_{0}$ the trivial character. Let $c: \hat{\Gamma}_{1} \rightarrow \hat{\Gamma}$ be a cross-section and let $X$ be the image of $c$ in $\hat{\Gamma}$. There is an isometry of $L^{2}(\hat{\Gamma})$ with $L^{2}\left(\hat{\Gamma}_{1}\right) \otimes C^{d}$ given by sending $\hat{g} \in L^{2}(\hat{\Gamma})$ to the vector of functions

$$
\left(\hat{g}(\omega), \hat{g}\left(\omega+\chi_{1}\right), \ldots, \hat{g}\left(\omega+\chi_{d-1}\right)\right.
$$

for $\omega \in X$.

The conditions of Lemma 3.1 can be reformulated to read:

(i) the $\Gamma_{1}$-translates of a $g \in D_{0}$ form an orthonormal set if

$$
\sum_{j}\left|\hat{g}\left(\omega+\chi_{j}\right)\right|^{2}=1
$$

for all $\omega \in X$ and hence for all $\omega \in \hat{\Gamma}_{1}$.

(ii) $g, g_{1} \in D_{0}$ span orthogonal $\Gamma_{1}$-modules if

$$
\sum_{j} \overline{\hat{g}\left(\omega+\chi_{j}\right)} \hat{g}\left(\omega+\chi_{j}\right)=0 \text {. }
$$

The existence of a $(\Gamma, \delta)$-wavelet $\psi_{1}, \ldots \psi_{d-1}$ is equivalent to the existence of a $d \times d$ unitary matrix valued function $M$ on $\hat{\Gamma}$ whose first column is the vector

$$
\left(h(\omega), h\left(\omega+\chi_{1}\right), \ldots, h\left(\omega+\chi_{d-1}\right)\right)
$$

and whose remaining columns are vectors

$$
\left(\hat{g}_{j}(\omega), \hat{g}_{j}\left(\omega+\chi_{1}\right), \ldots, \hat{g}_{j}\left(\omega+\chi_{d-1}\right)\right)
$$

for $j=1,2, \ldots d-1$. The relationship is simply that these columns define functions $g_{1}, g_{2}, \ldots g_{d-1}$ on $\Gamma$ with $\psi_{j}=\delta\left(g_{j}(\phi)\right)$. As a corollary of this we note the following extension of a theorem of Grochenig [g].

Proposition 3.3. If the function from $\hat{\Gamma}$ to $\mathbb{C}^{d}$ given by (3.2) does not map onto the $d-1$ sphere in $\mathbb{C}^{d}$ then there exists a unitary $d \times d$ matrix valued function $M$ whose first column is given by (3.2) which is as smooth as $\hat{h}$ (but not necessarily of compact support as a function on $\Gamma$ ). 
The proof is identical to that in [g]. Finally we note the approach in [s]. In our context this amounts to starting with a matrix valued function $M$ on $\hat{\Gamma}$ with first column given by (3.2) which is bounded away from zero and then applying a Gram-Schmidt process to the columns to produce a unitary matrix. This process preserves smoothness but not compact support properties. We will not describe this process in detail here but refer the reader to $[\mathrm{s}]$.

In our discussion we have not addressed the question of the existence of a scaling vector. This is the question which has received most attention in the literature and clearly the existence of these objects needs to be addressed in our general setting. Finally, Theorem 2 suffers from its lengthy and technical hypotheses. It would be interesting to discover other theorems, guaranteeing the existence of smooth wavelets, that have dissimilar assumptions to those of Theorem 2. The case where $\Gamma$ is non-abelian is however clearly a situation where more sophisticated techniques are called for (cf [1]).

\section{References}

[d] Daubechies, I., Orthonormal bases of compactly supported wavelets, Comm. Pure Appl. Math., 41 (1988), 909-928.

[dx] Dixmier, J., Von Neumann Algebras, North-Holland, Amsterdam, New York, Oxford, 1981.

[g] Grochenig, K., Analyse multi-echelles et bases d'ondelettes, C. R. Acad. Sci. Paris, 305 (1987), 13-15.

[1] Lemarie, P. G., Bases d'ondelettes sur les groupes de Lie stratifies, Bull. Math. Soc. France, 117 (1989), 211-233.

[m] Mandelbrojt, S., Series de Fourier et Classes Quasi-analytiques de Fonctions, GauthierVillars, Paris, 1935.

[ma1] Mallat, S., Multi-resolution approximations and wavelet orthonormal bases of $L^{2}(\mathbf{R})$, Trans. Amer. Math. Soc., 315 (1989), 69-87.

[ma2] —, A Theory for Multiresolution Signal Decomposition: The Wavelet Representation, IEEE Trans. Pat. Anal., 11 (1989), 674-693.

[me] Meyer, Y., Ondelettes, Hermann, Paris, 1990.

[rie] Rieffel, M., Induced representations of $C^{*}$-algebras, Adv. Math., 41 (1974), 176-258.

[s] Stöckler, J., Multivariate Wavelets, Wavelets: A tutorial in theory and applications, Academic Press, 1992. 
